\title{
Pleural effusion in polycystic kidney and liver disease
}

\section{Jessica Taylor ${ }^{1}$ and Adel Ekladious ${ }^{2 *}$}

${ }^{1}$ Royal Perth Hospital, Victoria Square Perth, Australia

${ }^{2}$ Faculty of Health and Medical Signs, University of Western, Australia

44 years old woman with polycystic liver and kidney disease presented to the emergency department with left pleuritic chest pain and recurrence of left upper quadrant pain. She presented 4 months ago with abdominal pain due to acute hepatic cyst rupture, with large free abdominal fluid demonstrated on CT (Panel A). The gross hepatomegaly was clinically evident, and laboratory tests showed mild elevation in ALP, GGT and normal renal function. CT chest and abdomen revealed massive hepatomegaly with innumerable large cysts (Panel B), mild free fluid in the pelvis, and large left pleural effusion (Panel C).

The women with polycystic kidney and liver disease are more likely to develop multiple, large cysts because of the growth stimulating effect of oestrogen.

An intercostal catheter was inserted and fluid analysis was consistent with transudate effusion, suggesting hepatic hydrothorax.

The literature review showed most pleural effusion in the context of polycystic liver disease are exudative, the development of hydrothorax in these patients are due to fenestration. In the tendinous portion of the diaphragm, positive intra-abdominal pressure and negative intra pleural pressure facilitates transmission of the fluid in the abdomen into pleural cavity through the congenital defects.

There are rare cases where the patients will develop pleural effusion in the absence of ascites due to polycystic liver disease and portal hypertension [1-3].

This case has three rare features, Polycystic disease of the liver is not common disease,

Left sided effusion in the absence of frank ascites, heart failure, pneumonia,

Nephrotic syndrome, anasarca, hypalbuminaemia or lymphatic obstruction is very rare,

The third rarity is to develop transudative and not exudative effusion after rupturing of the cyst is extremely rate

our patient has recurrent admissions to the hospital admission effusion abdominal pain due to ruptured cysts and massive pleural effusion, the patient listed for liver transplant as a curative treatment.

Copyright: (C2021 Taylor J. This is an open-access article distributed under the terms of the Creative Commons Attribution License, which permits unrestricted use, distribution, and reproduction in any medium, provided the original author and source are credited.
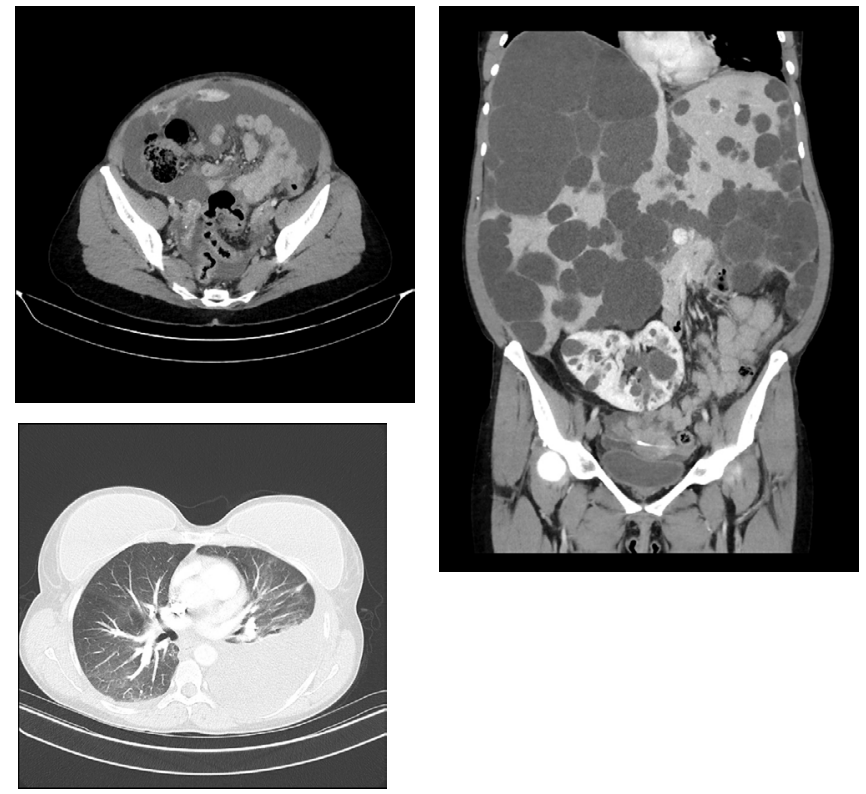

Figure 1. Left sided pleural effusion

\section{References}

1. Bistritz L, Tamboli C, Bigam D, Bain D, Bain VG (2005) Polycystic liver disease: Experience at teaching hospital. AM J Gastroentrology 100(10): 2212-2217. [Crossref]

2. Newman KD, torres VE, Rakela J, Nagorney DM (1990) Treatment of highly Symptomatic polycystic liver disease. Ann Surg 212(1): 30-37. [Crossref]

3. Zenda T, Miymoto S, Abuchi HM (1998) Detection of diaphragmatic defect as a cause of severe Murata S,Mabuchi, detection ofdiaphragmatic defect. Am J as a cause of severe hydrothorax with magnetic resonance imaging imaging, Aam J Gastroenterol 93: 2288-2289.

${ }^{\star}$ Correspondence to: Adel Ekladious, Faculty of Health and Medical Signs, University of Western, Australia, E-mail: ekladiou@hotmail.com

Received: January 03, 2021; Accepted: February 05, 2021; Published: February 08,2021 